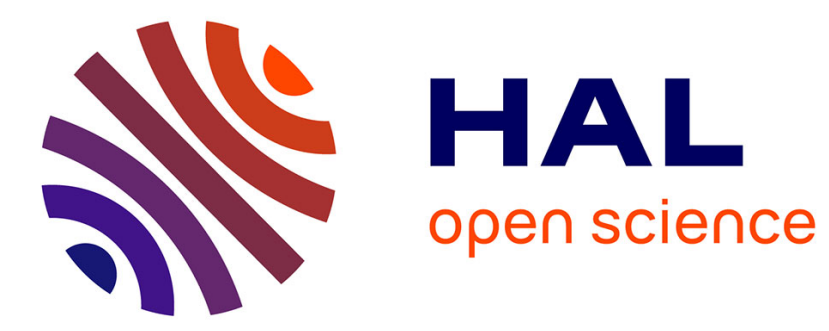

\title{
Arc Appearance and Cathode Spot Distribution in a Long-Gap High-Current Vacuum Arc Controlled by an External Axial Magnetic Field
}

B. Tezenas Du Montcel, P Chapelle, C. Creusot, A. Jardy

\section{- To cite this version:}

B. Tezenas Du Montcel, P Chapelle, C. Creusot, A. Jardy. Arc Appearance and Cathode Spot Distribution in a Long-Gap High-Current Vacuum Arc Controlled by an External Axial Magnetic Field. IEEE Transactions on Plasma Science, 2018, 46 (10), pp.3722-3730. 10.1109/TPS.2018.2858284 . hal-02106978

\section{HAL Id: hal-02106978 \\ https://hal.science/hal-02106978}

Submitted on 13 Jan 2022

HAL is a multi-disciplinary open access archive for the deposit and dissemination of scientific research documents, whether they are published or not. The documents may come from teaching and research institutions in France or abroad, or from public or private research centers.
L'archive ouverte pluridisciplinaire HAL, est destinée au dépôt et à la diffusion de documents scientifiques de niveau recherche, publiés ou non, émanant des établissements d'enseignement et de recherche français ou étrangers, des laboratoires publics ou privés. 


\title{
Arc appearance and cathode spot distribution in a long gap high-current vacuum arc controlled by an external axial magnetic field
}

\author{
B. Tezenas du Montcel, P. Chapelle, C. Creusot, and A. Jardy
}

\begin{abstract}
An experimental study of a high-current vacuum arc (VA) generated between two static CuCr25 contacts spaced 20 or 30 mm apart was conducted to characterize the arc appearance and the cathode spot (CS) distribution. The arc was ignited from the lateral surface of the cathode and controlled using an external axial magnetic field. Under the investigated experimental conditions, three distinct arc modes have been observed: multiple arc, diffuse and diffuse columnar modes. The latter appeared at low $\mathbf{B}_{\mathrm{AMF}} / \mathbf{I}_{\mathrm{arc}}$ values, typically below $4 \mathrm{mT} / \mathrm{kA}$. The temporal evolutions of the spot spatial distribution and of the associated distribution of the current density were analyzed by processing high-speed video images of the cathode. Various types of distribution depending mainly on current were identified. At low currents (up to $13 \mathrm{kA}$ ), CS distribution covered only a fraction of the cathode surface. At intermediate currents (in 17.2 - 31.1 kA range), CSs were present on the whole circumference of the cathode and the CS distribution included a closed region without spot. The latter was progressively filled by CSs, yet it totally disappeared before the end of arcing only when its location was off-centered with respect to the cathode axis. At high currents (up to $36.3 \mathrm{kA}$ ), the whole cathode surface was occupied by CSs at current peak. The azimuthally averaged radial distribution of the current density was found to be relatively uniform in the regions occupied by CSs. An average spot current of $36.5 \mathrm{~A} \pm 2.5 \mathrm{~A}$ has been estimated.
\end{abstract}

Index Terms - Vacuum arc, axial magnetic field, long gap, arc appearance, cathode spot distribution, experimental study.

\section{INTRODUCTION}

$\mathrm{V}$ ACUUM interrupter is nowadays the most widely used switching technology for medium voltage applications, whereas $\mathrm{SF}_{6}$ interrupter is the dominant technology in high voltage applications. In an attempt to develop $\mathrm{SF}_{6}$ free alternative circuit breakers owing to environmental considerations, research efforts are currently being made to increase the voltage rating of vacuum interrupters [1,2]. As a result, the behavior of a vacuum arc (VA) for contact gap above 10 mm needs to be better understood. Studies on this latter point are still limited. Most of the published papers on this topic are focused on vacuum arcs controlled with an axial magnetic field (AMF). Studies have been carried out for the case of large (10-40 mm) and very large (40-60 mm) contact gaps. Cheng et al. [3] investigated VAs with current up to $14.25 \mathrm{kA}$ between 50 mm diameter electrodes spaced $40 \mathrm{~mm}$ apart. Instable arc modes for the highest currents were mentioned for this configuration. Xiu et al. [4] have studied into more detail these instabilities for VAs with current up to $11 \mathrm{kA}$ for 75-100 mm diameter electrodes with gaps in 20$60 \mathrm{~mm}$ range. They classified them into two groups (normal and spiral distortions) and correlated their apparition with a magnetohydrodynamic criterion. Recently, Wang et al. [5] have studied the increase of the plasma constriction near the anode, within a 10 to $40 \mathrm{~mm}$ contact gap range, for applied current up to $28 \mathrm{kA}$ and using either $90 \mathrm{~mm}$ diameter cup-shaped electrodes or $110 \mathrm{~mm}$ diameter coil type electrodes. Besides the behaviour of the interelectrode plasma, the appearance of VAs at the anode has also been investigated by a few researchers. Cheng and Wang [6] have analyzed the anode phenomena for VAs with very large gaps for $100 \mathrm{~mm}$ diameter single coil type electrodes. Considering drawn arcs between $100 \mathrm{~mm}$ diameter 2/3 coil type electrodes, Yao et al. [7] have investigated, for large and very large gaps, the various modes of existence of the arc at the anode (passive anode, footpoint and anode spot) according to the current and arc length.

In VA, the plasma is emitted at the cathode surface by tiny, mobile cathode spots (CSs). Their motion and spatial distribution have a significant influence on the arc aspect and the plasma parameters [8]. CS motion is influenced by numerous factors, such as surface conditions, magnetic field intensity and plasma density $[9,10]$. To determine the spatial distribution of CSs over the cathode surface, Afanas'ev et al. [11] developed an original processing method, based on post-treatment of cathode images (previously) recorded with a high-speed camera. In this method, the position of CSs is extracted by filtering light intensity

Manuscript received September 13, 2017; accepted July 9, 2018

Corresponding Author: P. Chapelle

B. Tezenas du Montcel is with the Institut Jean Lamour, UMR 7198, Université de Lorraine/CNRS, 2 allée André Guinier, BP 50840, 54011 Nancy Cedex, France and also with the SuperGrid Institute, 23 rue Cyprian, BP 1321, 69611 Villeurbanne, France.

P. Chapelle and A. Jardy are with the Institut Jean Lamour, UMR 7198, Université de Lorraine / CNRS (e-mail: pierre.chapelle@ univ-lorraine.fr).

C. Creusot is with the SuperGrid Institute. 
contours. Moreover, the method allows estimating the distribution of current density at the cathode from the reconstructed CS distribution. Up to now, the method was employed to investigate VAs with small gap spacing (from 2 to $16 \mathrm{~mm}$ ) with a current of 3 to $25 \mathrm{kA}$ and contacts of $30-50 \mathrm{~mm}$ diameter [10, 12-14]. Moreover, it should be noted that all of these studies were performed for static contacts, the arc triggering being carried out with an auxiliary electrode placed at the cathode center.

This study aims to investigate experimentally the behavior of AMF controlled VAs with large gap distance (20 mm or $30 \mathrm{~mm})$ triggered with an auxiliary electrode placed close to the cathode outer diameter. First, arc visualization results are presented, describing arc mode and the interelectrode plasma appearance. Then, the temporal evolutions of CS distribution and current density are described, applying a similar spot detection method as proposed by Afanas'ev et al. [11].

\section{EXPERIMENTAL}

\section{A. Setup}

Experiments have been conducted in a demountable vacuum chamber with a pumping system capable of maintaining a vacuum ranging from $10^{-7} \mathrm{~Pa}$ to $10^{-5} \mathrm{~Pa}$, as depicted in Fig. 1. A pair of static electrodes was disposed inside the chamber with a controlled gap. The contacts were $70 \mathrm{~mm}$ diameter hollow cylinders of OFHC copper, surmounted by $2 \mathrm{~mm}$ thick CuCr25 alloy disks. A sixteen turns Helmholtz coil pair was installed outside the chamber to generate an AMF in the contact gap region. The arc was triggered with the help of an auxiliary electrode located close to the cathode outer diameter and connected to a HighVoltage $(20 \mathrm{kV})$ pulsed power supply. The arc and Helmholtz coils were powered from two independent $50 \mathrm{~Hz}$ generators. Current and voltage were measured at different locations of the electrical circuit. Four shunts were installed to measure the injected current $\mathrm{I}_{\mathrm{BT}}$, the output current $\mathrm{I}_{\text {arc }}$, the leakage current $\mathrm{I}_{\text {leak }}$ and the current in the Helmholtz coils $\mathrm{I}_{\text {coils. The arc voltage }}$ $\mathrm{U}_{\text {arc }}$ was obtained by measuring the potential difference upstream and downstream the chamber using two voltage dividers. Measured data were recorded on an acquisition bay with a sample frequency of $1 \mathrm{MHz}$. The electric arc was filmed with a highspeed camera Photron SA5 with an exposure time of $369 \mathrm{~ns}$ and a frame rate of 15000 or 20000 frames/s.

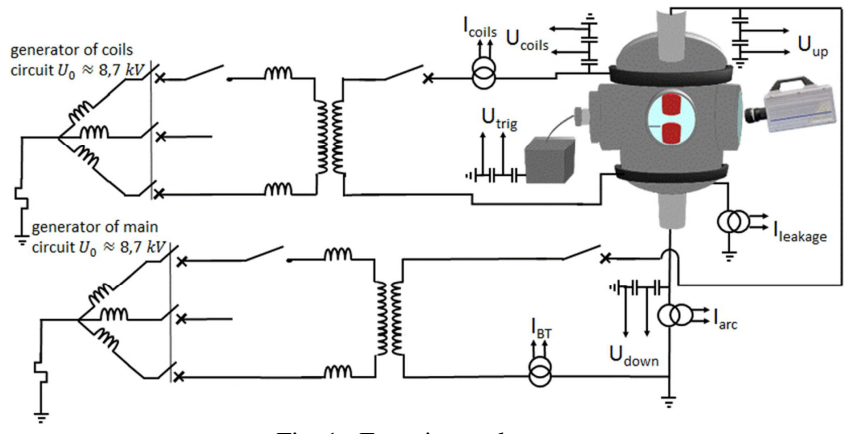

Fig. 1. Experimental setup.

Fig. 2 shows the radial profiles of the AMF intensity and the time delay between the AMF intensity and the coil current measured at mid-distance between the two contacts (spaced $25 \mathrm{~mm}$ apart) using a Hall probe placed on a mobile support. It can be seen that there is an important effect of the contacts upon the two parameters. The radial profile of the AMF has a saddle shape with values in 9.6-17.1 mT/kA range inside the contact gap region, while the time delay between the AMF intensity and the coil current has a bell-shaped profile with values in 3.4-5.2 ms range inside the contact gap region.

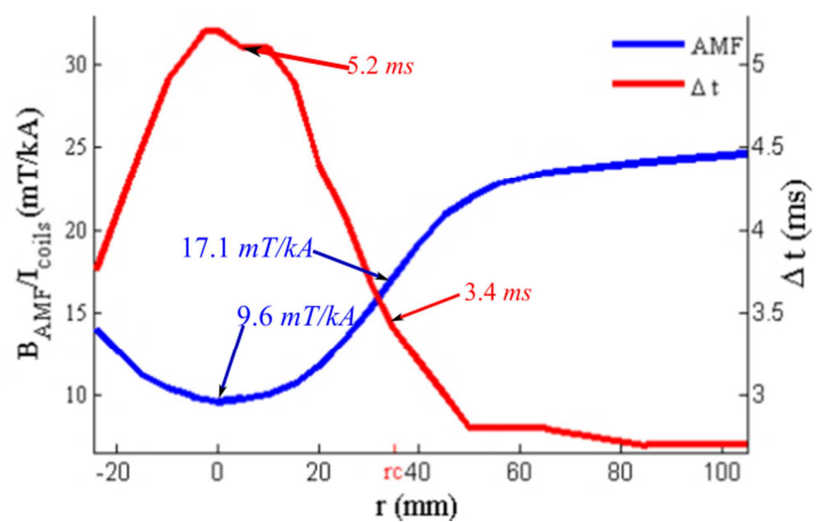

Fig. 2. Radial profiles of the AMF intensity and the time delay $\Delta t$ between $B_{\mathrm{AMF}}$ and $\mathrm{I}_{\text {coils }}$ measured at mid-distance between the two contacts. The symbol rc on the $\mathrm{x}$-axis refers to the radius of the contacts. 
As illustrated on Fig. 3, the arc was triggered when the voltage between the contacts reached a peak value and interrupted after a half-wave of current. The current injection in the Helmholtz coils was triggered $3.4 \mathrm{~ms}$ before the arc ignition in order to synchronize the peak of arc current with the AMF peak at the edge of the cathode. Moreover the current half-wave in the coil had a duration of $14 \mathrm{~ms}$ to maintain an intense AMF between the contacts during most of the arc lifetime. The coil current was finally interrupted using an auxiliary circuit breaker.

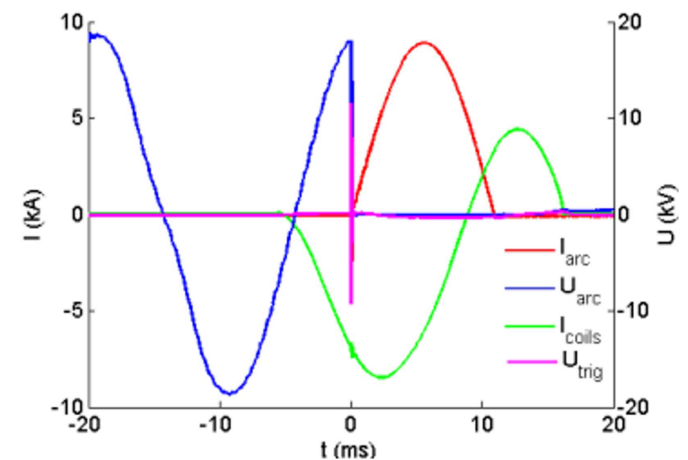

Fig. 3. Example of experimental procedure illustrating the synchronization of the arc and coil currents.

\section{B. Operating parameters}

The parameters used for the tests are presented in Table I. Exp. 1 to 7 were performed with a gap of 20 mm and with arc peak current values varying between $4.9 \mathrm{kA}$ and $43.3 \mathrm{kA}$. Exp. 8 to 11 were performed with a gap of $30 \mathrm{~mm}$ and with arc peak current values varying between $8.9 \mathrm{kA}$ and $24.9 \mathrm{kA}$. All experiments were realized with an AMF of about 143 mT oriented towards the cathode, except Exp. 7 during which the AMF was oriented towards the anode and its intensity was fixed at 269 mT. Note that Exp. 1-6 and 8-11 were realized one after the other with the same contacts whereas a new pair of similar contacts was used during Exp. 7.

TABLE I

GAP DISTANCE, ARC CURRENT, ARC DURATION AND AMF MAGNITUDE DURING EACH EXPERIMENT

\begin{tabular}{cccccc}
\hline \hline Exp. & $\begin{array}{c}\mathrm{L}_{\text {arc }} \\
(\mathrm{mm})\end{array}$ & $\begin{array}{c}\mathrm{I}_{\text {arc }} \\
(\mathrm{kA})\end{array}$ & $\begin{array}{c}\mathrm{T}_{\text {arc }} \\
(\mathrm{ms})\end{array}$ & $\begin{array}{c}\mathrm{B}_{\text {AMF }} \\
(\mathrm{mT})\end{array}$ & $\begin{array}{c}\mathrm{B}_{\text {AMF }} / \mathrm{I}_{\text {arc }} \\
(\mathrm{mTT} / \mathrm{kA})\end{array}$ \\
\hline 1 & 20 & 4.9 & 6.2 & 143 & 29.2 \\
2 & 20 & 17.2 & 13.7 & 143 & 8.3 \\
3 & 20 & 25.7 & 11.8 & 143 & 5.6 \\
4 & 20 & 26.7 & 9.9 & 143 & 5.4 \\
5 & 20 & 31.1 & 10.8 & 143 & 4.6 \\
6 & 20 & 36.3 & 11.7 & 143 & 3.9 \\
7 & 20 & 43.3 & 10.9 & -269 & 6.2 \\
8 & 30 & 8.9 & 10.9 & 146 & 16.1 \\
9 & 30 & 13 & 10.9 & 144 & 11 \\
10 & 30 & 18 & 10.9 & 143 & 7.9 \\
11 & 30 & 24.9 & 10.9 & 141 & 5.7 \\
\hline \hline
\end{tabular}

\section{Image processing method}

The image processing method employed to detect CSs on the cathode image is inspired by the work of Afanas'ev et al. [11].

The first step of the method consists in the detection, selection and density computing of iso-contours of light intensity on the image of the cathode front surface. The contours are selected according to their intensity, to eliminate the darkest contours, as well as according to their size, to reject too long and too short contours which cannot surround a single spot. Next, we calculate the centroid position of each selected contour and then the number of iso-contour centroid on each pixel. Above a certain threshold of iso-contour number, a pixel is marked and considered as a spot location. A limitation of this approach is that the same spot may end up to be detected on several adjacent pixels. To solve such a situation, only the centroid of each connected pixel region is conserved. Finally, the few false spots associated to a local minimum of light intensity, which may be detected after the complete processing procedure, are excluded based on an additional threshold filtering. The result of these successive operations is illustrated on Fig. 4. 


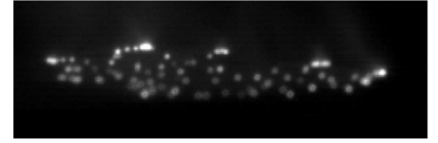

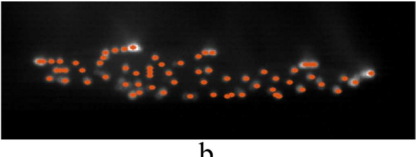

b

Fig. 4. (a) Example of cathode image, (b) the same image with cathode spots detected using the numerical treatment.

CS coordinates need then to be corrected to take into account the deformation of the cathode surface caused by the tilting of the camera. This can be done using a projective transformation relating the spot coordinates on the deformed image (x,y) to the spot coordinates on the rectified image $\left(\mathrm{x}^{\prime}, \mathrm{y}^{\prime}\right)[15]$ :

$$
\left\{\begin{array}{l}
x^{\prime}=\frac{h_{11} x+h_{12} y+h_{13}}{h_{31} x+h_{32} y+1} \\
y^{\prime}=\frac{h_{21} x+h_{22} y+h_{23}}{h_{31} x+h_{32} y+1}
\end{array}\right.
$$

The transformation involves eight coefficients $h_{i, j}$. Those coefficients were determined from four reference points taken from a $10 \mathrm{~mm}$-square reticle grid which was disposed after the tests on the front surface of the cathode and shot with the same viewing angle as the one used during the experiments.

The projective transformation is also applied to the points defining the contour of the cathode surface. Due to experimental uncertainties, the cathode contour obtained after this step is still elliptic with an eccentricity below 0.4 . Thus, a final correction step is needed to make the contour perfectly circular. The radial coordinate of the points located on the cathode contour, measured from the center of the ellipse obtained after the projective transformation, is replaced by the minor radius of the ellipse. Similarly, the radial coordinate $r_{\text {spot }}$ of a CS, defined with respect to the center of the ellipse obtained after the projective transformation, is replaced by the following expression $r_{\text {spot }} \sqrt{1-e^{2} \cos ^{2} \varphi_{\text {spot }}}$, where e is the eccentricity of the ellipse and $\varphi_{\text {spot }}$ is the spot angular position with respect to the major axis of the ellipse emanating from the center of the ellipse.

From the CS map, the distribution of the current density at the cathode was reconstructed by partitioning the cathode surface into Voronoi cells, each cell enclosing a single spot. The current density in each cell was considered to be inversely proportional to the cell area. Next, an azimuthally averaged radial profile of the cathode current density was determined. This profile was calculated using a harmonic mean formula (instead of an arithmetic mean) in order to be less sensitive to extreme values, which may occur when several CSs are located very close to each other, leading to very small Voronoi cells.

\section{ARC COLUMN}

In this section, we first describe the evolution of the arc appearance during the current wave for two different peak values with a gap of $20 \mathrm{~mm}$. Then, we detail the various arc modes observed for the range of conditions investigated in the present study.

Fig. 5 presents an example of the currents and arc voltage as a function of time measured during Exp. 3 and 6, which were carried out with the same gap length of $20 \mathrm{~mm}$ and with a peak current of $25.7 \mathrm{kA}$ and $36.3 \mathrm{kA}$ respectively. The AMF intensity and the overall plasma luminosity, defined as the integration of light intensity in the contact gap region are also represented on this figure. For both cases, a leakage current (up to $3 \mathrm{kA}$ ), that matches with the difference between $\mathrm{I}_{\text {arc }}$ and $\mathrm{I}_{\mathrm{BT}}$, exists during most of the first half wave of the arc current, meaning that a fraction of CSs is located on the walls of the chamber, while no significant leakage current is detected during the second half wave of the arc current. As will be seen below, the existence of a significant leakage current during the first half-wave of current is due (partly if not completely) to the location of spots on the lateral surface of the cathode immediately after arc ignition. Those spots may cause significant leaks of plasma out of the interelectrode space, which may enhance the apparition of CSs on the walls of the chamber. In contrast, during the second halfwave of current, most of the CSs are located on the front surface of the cathode, reducing the current leaks. A very high spike of the arc voltage is observed at the instant of ignition of the arc. Afterward, the variations of $U_{\text {arc }}$ are heavily influenced by the impedance of the experimental circuit. 


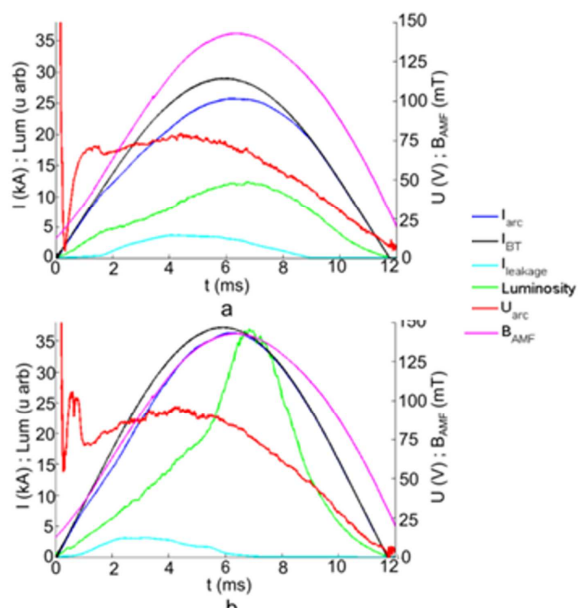

Fig. 5. Time evolutions of $\mathrm{I}_{\mathrm{arc}}, \mathrm{I}_{\mathrm{BT}}, \mathrm{I}_{\mathrm{leak}}, \mathrm{U}_{\mathrm{arc}}, \mathrm{B}_{\mathrm{AMF}}$ and the overall plasma luminosity during Exp. (a) 3 and (b) 6 .

Fig. 6 shows images of the arc column every millisecond during Exp. 3 and 6. As seen in Fig. 6a, after arc ignition, CSs are localized on the edge of the cathode, covering only a fraction of the circumference. From 1 to $6 \mathrm{~ms}$, the spots spread from the edge to the center of the cathode covering almost completely the cathode surface at the peak current. Individual plasma jets produced by the spots are clearly visible in the near cathode region. As the current increases, the plasma jets mix progressively and form a relatively homogeneous interelectrode plasma, which is characteristic of an arc operating in a diffuse mode. From 7 to $11 \mathrm{~ms}$, during the current decrease phase, the luminosity of the interelectrode plasma decreases gradually. As seen in Fig. 5a, the overall luminosity of the plasma is roughly proportional to the arc current. As shown in Fig. 6b, the evolution of the arc appearance at higher current is significantly different. Until $4 \mathrm{~ms}$, the arc develops similarly to that observed in Fig. $6 \mathrm{a}$. However, contrary to Fig. 6a, from 5 to $8 \mathrm{~ms}$ the plasma distribution is strongly non uniform. A very bright plasma axial column emerges and grows from the central part of the contacts, surrounded by a diffuse plasma column. This corresponds to the so called diffuse columnar mode. The presence of this bright column is responsible for a strong increase of the overall luminosity of the plasma as observed in Fig. 5b. At $9 \mathrm{~ms}$, the arc appears diffuse again. Then the plasma becomes darker with the decrease of the arc current. While the central column luminosity decreases, the column radius changes only a little. Note that contrary to Fig. 6a, the CSs occupy completely the cathode surface at the current peak value. After the arc extinction, the optical inspection of the contacts revealed no traces of melting on the anode surface, which indicates that the anode remained passive during arcing.
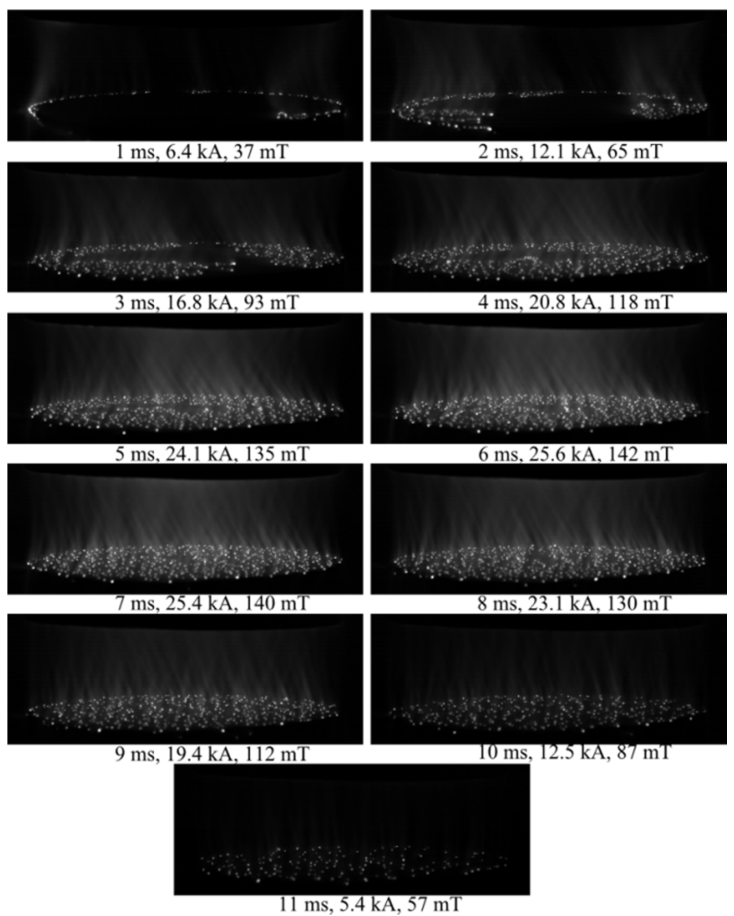

(a) 


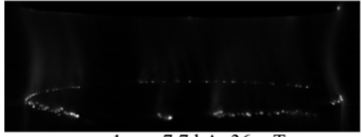

$1 \mathrm{~ms}, 7.7 \mathrm{kA}, 36 \mathrm{mT}$

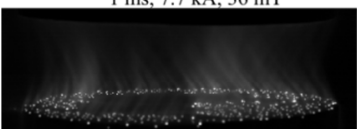

$3 \mathrm{~ms}, 22.0 \mathrm{kA}, 91 \mathrm{mT}$

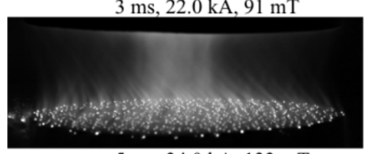

$5 \mathrm{~ms} .34 .0 \mathrm{kA} .133 \mathrm{mT}$

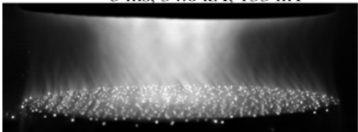

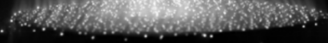

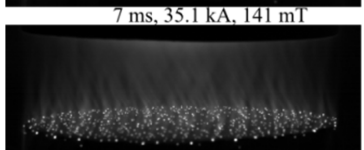

$9 \mathrm{~ms}, 23.8 \mathrm{kA}, 112 \mathrm{mT}$

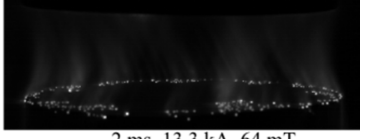

$2 \mathrm{~ms}, 13.3 \mathrm{kA}, 64 \mathrm{mT}$

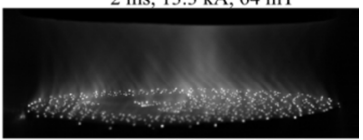

$4 \mathrm{~ms}, 28.8 \mathrm{kA}, 116 \mathrm{mT}$

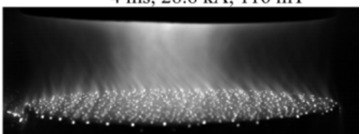

$6 \mathrm{~ms}, 36.2 \mathrm{kA}, 143 \mathrm{mT}$

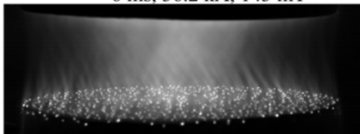

$\mathrm{ms}, 30.6 \mathrm{kA}, 129 \mathrm{mT}$

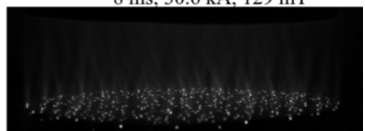

$10 \mathrm{~ms}, 15.4 \mathrm{kA}, 88 \mathrm{mT}$

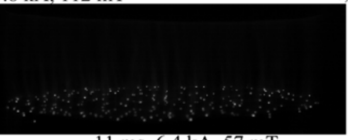

(b)

Fig. 6. Arc image every millisecond during Exp. (a) 3 and (b) 6 .

Fig. 7 and Fig. 8 show the arc appearance at the peak of current for all the performed experiments. Fig. 7 refers to a gap of 20 $\mathrm{mm}$, while Fig. 8 refers to a gap of $30 \mathrm{~mm}$.
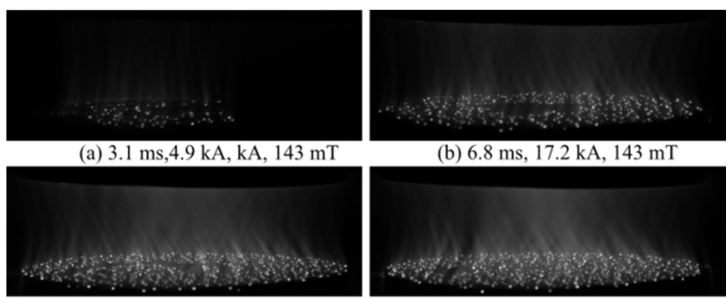

(b) $6.8 \mathrm{~ms}, 17.2 \mathrm{kA}, 143 \mathrm{mT}$
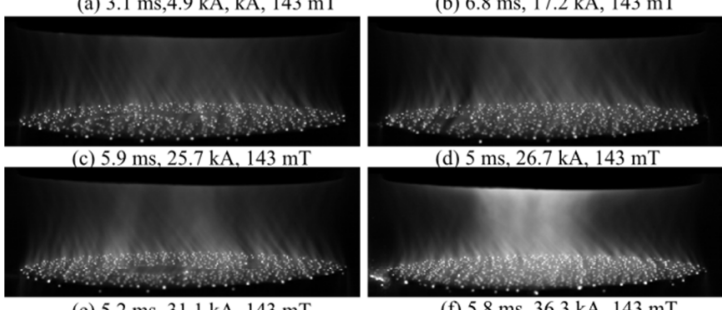

(d) $5 \mathrm{~ms}, 26.7 \mathrm{kA}, 143 \mathrm{mT}$

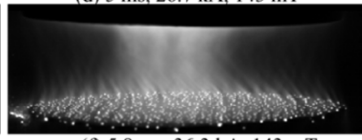

(e) $5.2 \mathrm{~ms}, 31.1 \mathrm{kA}, 143 \mathrm{mT}$ (f) $5.8 \mathrm{~ms}, 36.3 \mathrm{kA}, 143 \mathrm{mT}$

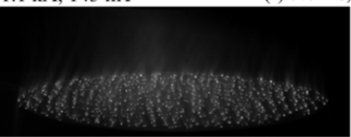

(g) $5.5 \mathrm{~ms}, 43.3 \mathrm{kA}, 269 \mathrm{mT}$

Fig. 7. Arc image at the current peak under a gap of $20 \mathrm{~mm}$. Exp. (a) 1, (b) 2, (c) 3, (d) 4, (e) 5, (f) 6 and (g) 7 .

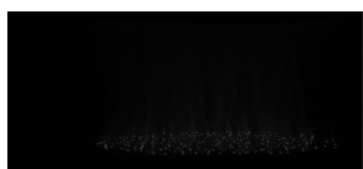

(a) $5.5 \mathrm{~ms}, 8.9 \mathrm{kA}, 146 \mathrm{mT}$
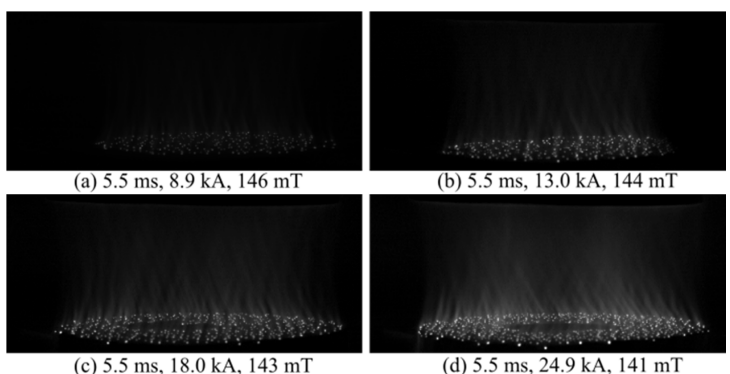

(b) $5.5 \mathrm{~ms}, 13.0 \mathrm{kA}, 144 \mathrm{mT}$

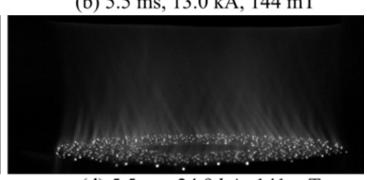

(d) $5.5 \mathrm{~ms}, 24.9 \mathrm{kA}, 141 \mathrm{mT}$

Fig. 8. Arc image at the current peak under a gap of $30 \mathrm{~mm}$. Exp. (a) 8, (b) 9, (c) 10 and (d) 11 .

When the gap is positioned at $20 \mathrm{~mm}$, essentially three different arc modes can be observed. For currents lower than $4.9 \mathrm{kA}$ 
(Fig. 7a), the arc operates in a multiple arc mode. In this mode, the plasma jets emitted by the CSs do not overlap. At currents higher than $17.2 \mathrm{kA}$ (Fig. 7b-e), the arc becomes diffuse. The mixing of the plasma jets produces a quite uniform plasma. Moreover, with the current increase, the mixing of the plasma jets tends to take place closer to the cathode. Finally, for current higher than $36.3 \mathrm{kA}$ (Fig. 7f), the arc appears as a diffuse columnar arc, with the formation of a bright plasma column in the central part of the contacts. Note that, while the arc current in Exp. 7 (Fig. 7g) was higher compared to that in Exp. 6 (Fig. 7f), the arc remains diffuse in Exp. 7 because the applied AMF is stronger. In Figure 7b-e, a counterclockwise inclination of the plasma jets is clearly visible. The inclination becomes more pronounced when the current increases. As discussed by Jia et al. [16], the flow direction of the jets is dependent on that of the composite magnetic field resulting from the superposition of the AMF and the azimuthal self-generated magnetic field. The inclination of this composite magnetic field gets stronger when the current increases, giving a more pronounced inclination of the jets. In accordance with this explanation, it is observed that the plasma jet inclination becomes clockwise in Exp. 7 (Fig. 7g) due to the reversal of the AMF direction.

When the gap is positioned at $30 \mathrm{~mm}$, only two arc modes are observed. The multiple arc mode is observed again at low current (Fig. 8a), while the arc is diffuse at currents higher than $13 \mathrm{kA}$ (Fig. 8b-d). In contrast to the experiments with a $20 \mathrm{~mm}$ gap, the arc exhibits no diffuse columnar mode. This may be related to lower values of the arc current investigated for a $30 \mathrm{~mm}$ gap (24.9 kA instead of $36.3 \mathrm{kA}$ under a similar AMF intensity). The lowest value reached by the ratio $\mathrm{B}_{\mathrm{AMF}} / \mathrm{I}_{\text {arc }}$ for a $30 \mathrm{~mm}$ gap is $5.7 \mathrm{mT} / \mathrm{kA}$. This value remains larger than the one for Exp. 6 with a $20 \mathrm{~mm}$ gap, during which a diffuse columnar arc mode was observed. Note that besides the influence of the ratio $\mathrm{B}_{\mathrm{AMF}} / \mathrm{I}_{\mathrm{arc}}$ we cannot exclude effects related to the increase of the gap length on the arc operating mode. As it was observed for a $20 \mathrm{~mm}$ gap, the plasma jets exhibit again a counterclockwise inclination, which gets stronger with the rise of the current.

Fig. 9 illustrates the evolution of the arc mode observed during Exp. 5 to 7 as a function of the instantaneous current and AMF magnitude. As the identification of the transition between the multiple arc mode and the diffuse arc mode is not straightforward (and based on visual criterion), the boundary between these two modes is not represented in Fig. 9. This figure suggests a linear relationship between the critical current and the critical AMF magnitude separating the existence domain of the diffuse mode and the diffuse columnar mode:

$$
I_{\text {arc }}=0.1\left[k A \cdot m T^{-1}\right] B_{A M F}+17[k A]
$$

This criterion is close to that deduced from the diagram presented by Schade and Shmelev (cf. Fig. 1 in [17]) given for 55 mm diameter copper contacts spaced $10 \mathrm{~mm}$ apart. It is also interesting to note that the curves relative to Exp. 5 and 7 remain below the derived boundary between the diffuse and diffuse columnar modes, in accordance with the observations made on the arc images.

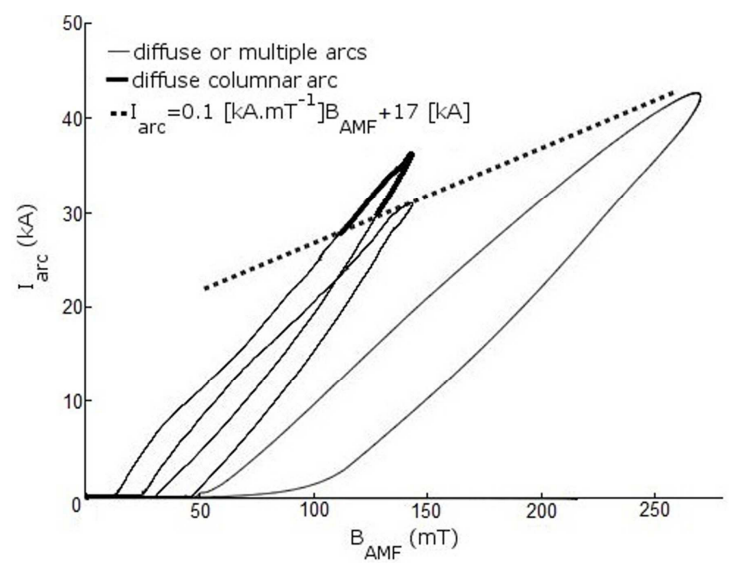

Fig. 9. Arc modes as a function of the instantaneous current and AMF magnitude during Exp. 5-7.

\section{CATHODE SPOTS}

In this section, the evolution of the spatial distributions of CSs and current density is studied on the cathode images posttreated with the previously presented CS detection method. Afterward, an estimation of the average current carried by a single spot is also presented.

\section{A. Cathode spot distribution}

First, the initial propagation of CSs after arc ignition is described. As illustrated in Fig. 10 for the case of Exp. 3, because of the lateral trigger system, the first CSs appear on the lateral surface of the cathode. These spots exhibit a very fast anticlockwise rotation motion around the cathode under the influence of the AMF [9]. From about $100 \mu \mathrm{s}$, some CSs reach the front surface, emerging at various locations along the circumference of the cathode. These spots are much slower than the spots established on the lateral surface. They begin to effectively spread over the cathode surface from about $700 \mu$ s. Finally, CSs established on the lateral surface of the cathode disappear totally at around $1 \mathrm{~ms}$ for that particular experiment. 


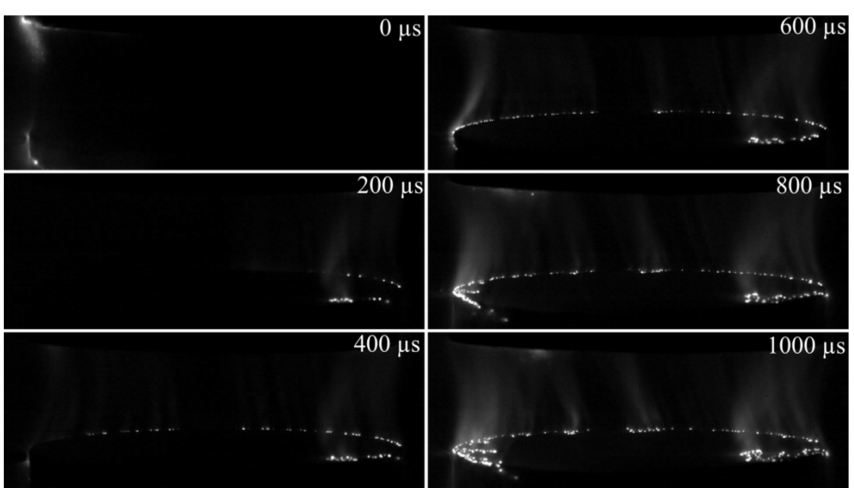

Fig. 10. Arc images every $200 \mu$ s during Exp. 3 just after arc ignition.

Fig. 11 presents typical time evolutions of the CS distribution that were observed depending on the arc peak current. Fig 11a shows that at low arc current (up to $13 \mathrm{kA}$ for the present conditions, i.e. in Exp. 1, 8 and 9) CSs form a compact group (with no enclosed empty region) which occupies only a reduced fraction of the cathode surface. At high current (above $36 \mathrm{kA}$ for the present conditions, i.e. in Exp. 6 and 7), as shown in Fig. 11d, CSs appear first along large sections of the circumference of the cathode, before spreading across the entire cathode surface. At the current peak, the whole cathode surface is occupied by the CSs. After the current peak, CSs tend to align along spiral arms originating from the center of the cathode. For intermediate values of current (from 17.2 kA to $31.1 \mathrm{kA}$ for the present conditions, i.e. in Exp. 2 to 5, 10 and 11), the CS distribution exhibits two different behaviors. Similarly to the case at high current, a CS empty region within the spatial distribution of CSs is formed during the rise of current as a result of the expansion of the CSs from the edge towards the center of the contact. The empty region may either be eventually totally filled and disappear as in Fig. 11b or persist, although its size decreases while the current rises, until the end of arcing as in Fig. 11c. As seen in Fig. 11 and confirmed by the observations made in the other experiments of Table I, it seems that the evolution of the CS empty region depends on its location with respect to the axis of symmetry of the cathode. The empty region eventually disappears in the case of an off-centered location, whereas it remains always present when its location is relatively centered.

Note that in all cases, during the decrease of the arc current, the disappearance of CSs seems to happen homogeneously over the cathode surface. Our results do not reveal any noticeable effect of the gap length on the CS distribution. Moreover, the presence of a bright plasma column at the center of the contacts in Exp. 6 seems to have no influence on the CS distribution.
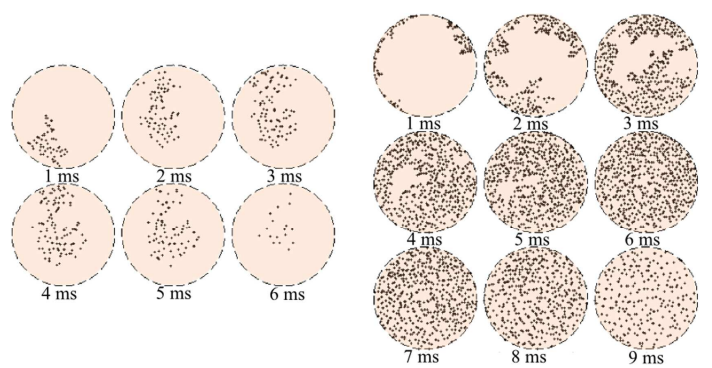

a
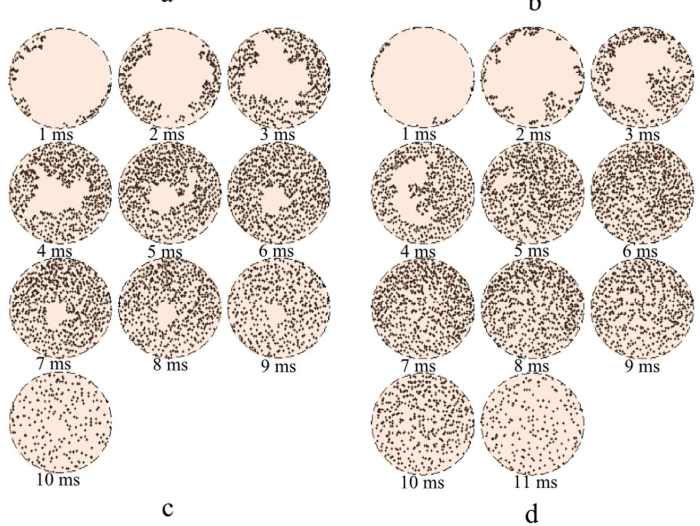

Fig. 11. Cathode spot distribution every millisecond during Exp. (a) 1 at $4.9 \mathrm{kA}$, (b) 4 at $26.7 \mathrm{kA}$, (c) 5 at $31.1 \mathrm{kA}$ and (d) 6 at $36.3 \mathrm{kA}$. 


\section{B. Cathode spot average current}

Fig. 12 shows an estimation of the average current carried by a CS, which was computed by dividing the instantaneous total arc current by the number of spots detected at that instant.

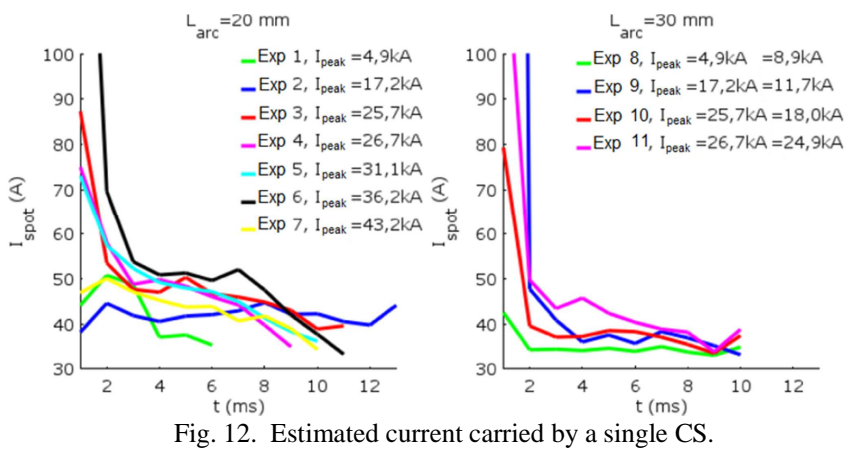

The quite high values of the spot current during the first milliseconds in some experiments can be explained by the presence of an important proportion of spots on the lateral surface of the cathode. Our processing method discards those spots. The number of spots being underestimated, the spot current is hence overestimated. Similarly, the number of spots may be slightly underestimated (hence the spot current will be slightly overestimated) during most of the arc lifetime, because of the ejection of spots out of the front surface of the cathode as a result of repulsive forces between spots. This effect is expected to weaken when the current decreases during the second half of the current wave, which explains the decreasing trend of the computed spot current values observed in Figure 12 and the converging trend of the curves associated to the different experiments. Therefore, it is believed that the most reliable spot current value are those at the end of arcing ( $1 \mathrm{~ms}$ before arc extinction), yielding to an average value of the current emitted by a spot of 36.5 $\pm 2.5 \mathrm{~A}$. When averaging the data presented in Fig. 12, the data of Exp. 2 has been excluded, since video images of the arc reveal an ejection of CSs out of the front surface still important at the end of arcing. This was interpreted as a consequence of a poor control of the arc due to the lower magnitude of the AMF at the end of arcing. AMF magnitude was lower at the end of arcing because arc ignition was exceptionally long during this experiment. The value of the average CS current obtained here is similar to the spot current measured by Yu et al. [18] for nanocrystalline $\mathrm{CuCr} 25$ contact material.

\section{Radial profile of the cathode current density}

Azimuthally averaged radial profiles of the cathode current density have been calculated from the position of CSs according to the procedure exposed in section II.C. Fig. 13 shows typical examples of those profiles for the same four experiments, whose CS distributions were presented in Fig. 11.

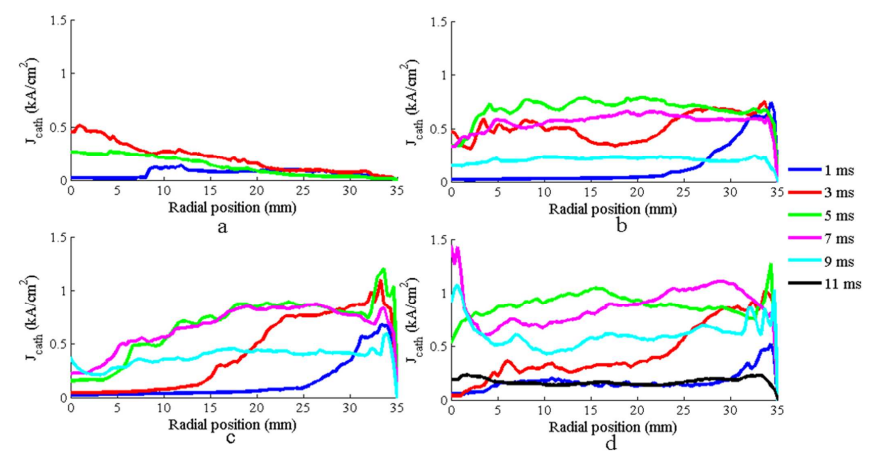

Fig. 13. Radial profiles of cathode current density for Exp. (a) 1, (b) 4, (c) 5, (d) 6.

At low current (Fig. 13a), except at the beginning of arcing, the current density decreases from the center to the edge of the cathode. At higher currents (Fig. 13b-d), because of the initial repartition of CSs on the edge of the cathode, the current density exhibits a peak at the cathode edge during the first milliseconds. Next, as the current increases and spots expand on the cathode surface, the peak widens and the front of current density moves towards the center of the cathode. It is interesting to note that the current density at the edge of the cathode shows only slight variation during the propagation of the front. At the current peak, when CSs occupy completely the cathode surface, such as in Exp. 4 and 6, the current density is approximately evenly distributed. In contrast, in Exp. 5, because of the persistence of a central CS empty region, the current density at the current peak is non uniform, with minimum values reached naturally near the center of the cathode. For all experiments, after the current 
peak, the current density tends to decrease uniformly over the surface. Note that the very large values of the current density observed close to the cathode center at $7 \mathrm{~ms}$ and $9 \mathrm{~ms}$ in Exp. 6 are believed to be computation artefacts, induced by a small number of spots averaged close to the center.

\section{Discussion}

CS motion (which is a random walk when no magnetic field is present) is strongly influenced by a magnetic field. In presence of a tangential magnetic field, it is well known that a CS is subjected to a directional "retrograde" motion, which is in the direction opposite to that given by the Lorentz force $\vec{J} \wedge \vec{B}_{t}$. The velocity of this directional motion depends on the material of the cathode and the magnetic field magnitude [16]. Superposing an axial magnetic field to the tangential magnetic field causes an additional angular drift with respect to the retrograde direction (called the Robson drift) [19]. The deflection angle $\theta$ was found to be proportional to the angle formed by the total magnetic field with the cathode surface [16]:

$$
\theta=\eta \operatorname{atan} B_{A M F} / B_{t}
$$

where the coefficient $\eta$ depends among others on the cathode material. In a high current VA, as many CSs are present simultaneously on the cathode surface, the retrograde motion of CSs under the combined effect of the self-magnetic fields generated by every spot results in self-repulsion between CSs and the expansion of CSs over the cathode surface [16].

The analysis of the CS distributions in section IV.A showed that the presence of an empty region within the CS distribution until the end of arcing, leading to only a partial occupation of the cathode surface, seems to correlate with the relatively centered location of the empty region, this characteristic being itself linked to the initial repartition of CSs along the cathode circumference. In the following, an interpretation of this result by examining the direction of the retrograde motion of the whole CSs is proposed. The direction of retrograde motion of CSs was calculated from the CS map presented above, following a similar approach to that employed by Jia et al. [20]. The tangential magnetic field produced by the current of a spot $\mathrm{i}$ at the location of a spot $\mathrm{n}$ is given by:

$$
\vec{B}_{i}(n)=\frac{\mu_{0}}{2 \pi} \frac{\left(I_{s p o t} \vec{e}_{z}\right) \wedge \vec{r}_{i-n}}{\left\|\vec{r}_{i-n}\right\|^{2}}
$$

where $\vec{r}_{i-n}$ is the displacement vector from the location of spot $\mathrm{i}$ to the location of spot $\mathrm{n}$. Then, the direction of the retrograde motion of spot $\mathrm{n}$ is given by:

$$
-\left(\frac{I_{\text {spot }}}{\pi R_{\text {spot }}^{2}} \vec{e}_{z}\right) \wedge \sum_{i=1}^{N_{\text {spot }}} \vec{B}_{i}(n)=\frac{\mu_{0}}{2 \pi^{2}} \frac{I_{\text {spot }}^{2}}{R_{\text {spot }}^{2}} \sum_{i=1}^{N_{\text {spot }}} \frac{\vec{r}_{i-n}}{\left\|\vec{r}_{i-n}\right\|^{2}}
$$

Due to the presence of an AMF, one needs also to take into account the angular deflection with respect to that direction caused by the AMF, the deflection angle being determined from equation (3). Based on the AMF magnitude profile presented in Figure 2 and considering a spot current of $36.5 \mathrm{~A}$, a spot radius of $50 \mu \mathrm{m}$ [21] and a typical value of the parameter $\eta$ equals to 0.8 (similar to that given for $\mathrm{CuCr} 30$ contacts in [22]), the direction of the retrograde motion of every CS can thus be calculated from the CS location map using equation (5). Fig. 14 shows an example of calculation results at three particular time instants during Exp. 4 and 5.

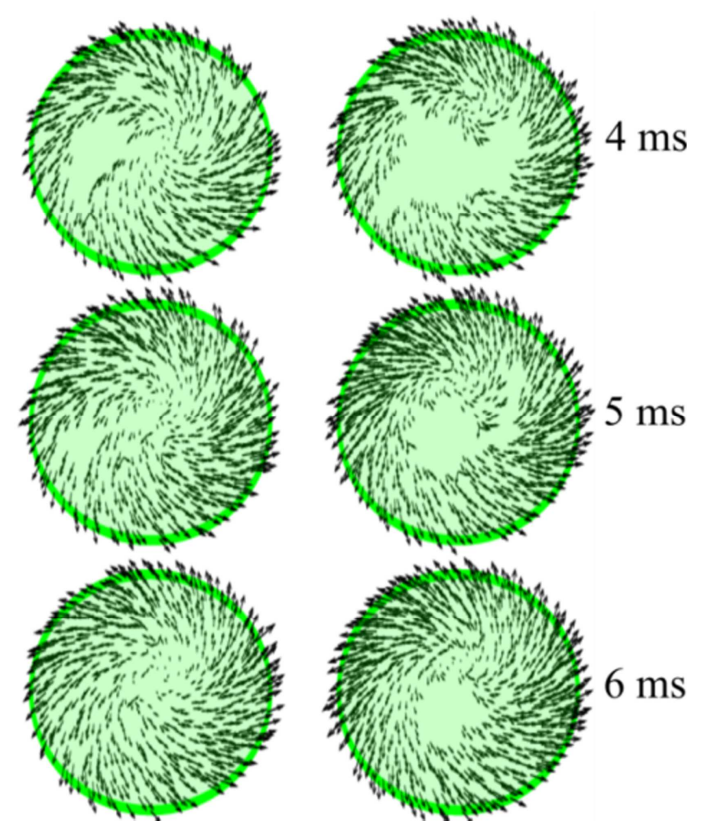


Fig. 14. Calculated direction of the retrograde motion with Robson drift of CSs during Exp. (left column) 4 and (right column) 5 at three particular time instants.

When the CS empty region is off-centered as in Exp. 4, it is observed that part of the CSs (essentially those close to the center of the cathode) have a motion directed towards the inside of the empty region and contribute to the decrease of the size of the empty region. Between 4 and $5 \mathrm{~ms}$, the area of the empty region falls by half. At $6 \mathrm{~ms}$, the empty region disappears and the cathode is fully occupied by CSs.

In contrast, when the CS empty region is relatively well centered as in Exp. 5, very few CSs exhibit a motion directed towards the inside of the empty region. Therefore, the empty region although decreasing in size remains present until the end of arcing. At $6 \mathrm{~ms}$, it represents about $10 \%$ of the cathode surface. Thus, the correlation observed between the persistence of the empty region inside the CS distribution and the centered position of the empty region in the case of moderate arc current may be explained by the CS motion direction, which does not favor the filling of the empty region.

\section{CONCLUSION}

VAs between $\mathrm{CuCr} 25$ static contacts spaced 20 and $30 \mathrm{~mm}$ apart with a range of peak current between 3.9 and $43.3 \mathrm{kA}$ and under a peak value of AMF of $143 \mathrm{mT}$ or $269 \mathrm{mT}$ were studied with a high speed camera to characterize the arc appearance and the CS distribution.

For a $20 \mathrm{~mm}$ gap, three arc modes were successively observed with increasing arc current, respectively a multiple arc mode, a diffuse arc mode and a diffuse columnar arc mode. The diffuse columnar arc mode occurred for a value of the $\mathrm{B}_{\mathrm{AMF}} / \mathrm{I}_{\mathrm{arc}}$ ratio of around $4 \mathrm{mT} / \mathrm{kA}$. For a $30 \mathrm{~mm}$ gap, the maximum current investigated was smaller (yielding to a minimum value of the $\mathrm{B}_{\mathrm{AMF}} / \mathrm{I}_{\mathrm{arc}}$ ratio of around $6 \mathrm{mT} / \mathrm{kA}$ ). No diffuse columnar arc mode was observed; the arc exhibiting either a multiple arc mode or a diffuse mode.

Various types of CS distribution have been identified, which are to some extent dependent on the specific arc ignition procedure on the lateral surface of the cathode used in the present work. At low currents, after reaching the front surface of the cathode, the CSs form a distribution without empty region, which covers only a fraction of the cathode surface. At moderate currents, a closed region unoccupied by CSs forms within the CS distribution during the development of the arc. This empty region, although decreasing in size, remains present until the end of arcing, when its location is centered with respect to the cathode axis. In contrast, the empty region was found to be eventually totally filled by CSs when its location is off-centered. An explanation of this behavior was proposed in terms of the direction of the retrograde motion of CSs located at the periphery of the empty region. When the current is further increased, due presumably to the increase of CS density, the cathode surface is always fully occupied by CSs at the current peak. No significant modification of the CS distribution with the gap length was observed. An average spot current of $36.5 \pm 2.5$ A was estimated. Our results suggest that the current density is relatively uniform in the regions occupied by CSs.

\section{ACKNOWLEDGMENT}

This project was supported by the French Government in the framework of the "Investments for the Future" programme under the project reference (ANE-ITE-002-01). The authors would like to thank F. Catanese, F. Metral and P. Robin for their help in the experimental work.

\section{REFERENCES}

[1] Y. Zhang, X. Yao, Z. Liu, Y. Geng, and P. Liu, "Axial magnetic field strength needed for a 126-kV single break vacuum circuit breaker during asymmetrical current switching", IEEE Trans. Plasma Sci., vol. 41, no. 6, pp. 1670-1678, May 2013.

[2] W. Hartmann, W. Haas, M. Römheld, and N. Wenzel, "AMF vacuum arcs at large contact separation," in Proc. XXIst Int. Symp. on Discharges and Electrical Insulation in Vacuum, Yalta, Crimea, 2004.

[3] S. Cheng, S. Xiu, J. Wang, and Z. Shen, "The discharge development and arc modes in vacuum at a long gap distance in vacuum interrupters," Plasma Sci. and Tech., vol. 9, no. 1, pp. 39-44, 2007.

[4] S. Xiu, Q. Li, G. Liu, and Z. Ye, "Study of distortion of a vacuum arc at a long-gap distance," Plasma Sci. and Tech., vol. 13, no. 2, pp. 188-193, 2011.

[5] L. Wang, L. Hu, J. Deng, X. Zhou, S. Jia, Z. Qian, X. Huang, and Z. Shi, "Experimental investigation on vacuum arc behaviors subjected to larger diameter cup-shaped and coil-shaped axial magnetic field electrode," IEEE Trans. Plasma Sci., vol. 43, no. 3, pp. 884-891, Mar. 2015.

[6] S. Cheng and J. Wang, "Study on high-current vacuum arc characteristics under self-generated axial magnetic field of contact at a long contact gap for highvoltage vacuum interrupters," IEEE Trans. Plasma Sci., vol. 37, no. 1, pp. 243-253, Jan. 2009.

[7] X. Yao, J. Wang, Y. Geng, and Z. Liu, "Anode mode diagram: a determination of opening displacement curve for a $126 \mathrm{kV}$ vacuum circuit breaker," in Proc. XXVIth Int. Symp. on Discharges and Electrical Insulation in Vacuum, Mumbai, India, 2014, pp. 189-192.

[8] A. M. Chaly, A. A. Logatchev, and S. M. Shkol'nik, "Cathode spot dynamics on pure metals and composite materials in high-current vacuum arcs," IEEE Trans. Plasma Sci., vol. 25, no. 4, pp. 564-570, Aug. 1997.

[9] B. Jüttner and I. Kleberg, "The retrograde motion of arc cathode spots in vacuum," J. Phys. D: Appl. Phys., vol. 33, no. 16, pp. 2025-2036, 2000.

[10]X. Song, Z. Shi, C. Liu, S. Jia, and L. Wang, "Influence of AMF on the expansion speed of cathode spots in high-current triggered vacuum arc," IEEE Trans. Plasma Sci., vol. 41, no. 8, pp. 2061-2067, Aug. 2013.

[11]V. P. Afanas'ev, A. M. Chaly, A. A. Logatchev, S. M. Shkol'nik, and K. K. Zabello, "Computer-aided reconstruction of cathode images obtained by high speed photography of high current vacuum arcs," IEEE Trans. Plasma Sci., vol. 29, no. 5, pp. 695-699, Oct. 2001.

[12]A. M. Chaly, A. A. Logatchev, K. K. Zabello, and S. M. Shkol'nik, "High-current vacuum arc appearance in nonhomogeneous axial magnetic field," IEEE Trans. Plasma Sci., vol. 31, no. 5, pp. 884-889, Oct. 2003. 
[13]A. M. Chaly, A. A. Logatchev, K. K. Zabello, and S. M. Shkol'nik, "High-current vacuum arc in a strong axial magnetic field," IEEE Trans. Plasma Sci., vol. 35, no. 4, pp. 939-945, Aug. 2007.

[14]Z. Shi, S. Jia, X. Song, Z. Liu, H. Dong, and L. Wang, "The influence of axial magnetic field distribution on high-current vacuum arc," IEEE Trans. Plasma Sci., vol. 37, no. 8, pp. 1446-1451, Aug. 2009.

[15]L. Jagannathan and C. V. Jawahar, "Perspective correction methods for camera-based document analysis," in Proc. Ist Int. Workshop on Camera Based Document Analysis and Recognition, Seoul, Korea, 2005.

[16]S. Jia, Z. Shi, and L. Wang, "Vacuum arc under axial magnetic fields: experimental and simulation research," J. Phys. D: Appl. Phys., vol. 47, pp. 1-24, 2014.

[17]E. Schade and D. L. Shmelev, "Numerical simulation of high-current vacuum arcs with an external axial magnetic field," IEEE Trans. Plasma Sci., vol. 31, no. 5, pp. 890-901, Oct. 2003.

[18]L. Yu, J. Wang, Y. Geng, G. Kong, and Z. Liu, "High-current vacuum arc phenomena of nanocrystalline CuCr25 contact material," IEEE Trans. Plasma Sci., vol. 39, no. 6, pp. 1418-1426, Jun. 2011.

[19] A. E. Robson, "The motion of a low-pressure arc in a strong magnetic field," J. Phys. D, Appl. Phys., vol. 11, no. 13, pp. 1917-1923, 1978.

[20]S. Jia, X. Song, Z. Shi, L. Wang, and X. Huo, "Investigations on the motion of high-current vacuum-arc cathode spots under a magnetic field," IEEE Trans. Plasma Sci., vol. 39, no. 6, pp. 1344-1348, Jun. 2011.

[21]B. Jüttner, "Cathode spots of electric arcs," J. Phys. D: Appl. Phys., vol. 34, no. 17, pp. R103-R123, 2001.

[22]A. M. Chaly and S. M. Shkol'nik, "Low-current vacuum arcs with short arc length in magnetic fields of different orientations. A review," IEEE Trans. Plasma Sci., vol. 39, no. 6, pp. 1311-1318, Jun. 2011. 